\title{
Universalização do acesso ao Sistema Único de Saúde no Brasil: desafios para a Atenção Primária à Saúde
}

\author{
Regina Maria Giffoni Marsiglia \\ Doutora em Ciência Política, Professora Adjunta, Faculdade de Ciências Médicas, Santa Casa de São Paulo, \\ Assistente Doutora, Pontifícia Universidade Católica de São Paulo, São Paulo, SP, Brasil
}

\begin{abstract}
Resumo: Introdução: Vinte e cinco anos após a promulgação da Constituição Federal de 1988 no Brasil, que definiu um Sistema Único de Saúde - SUS para o país, muitos são os avanços, mas permanecem grandes desafios. Objetivos: apresentar as bases teóricas e práticas da Atenção Primária à Saúde (APS); a experiência do Brasil e de São Paulo. Discutir a categoria acesso a serviços, as "portas de entrada" para o SUS; desafios para a universalização do acesso, a partir da Atenção Primária à Saúde. Metodologia: análise sobre resultados de pesquisas que investigaram diferentes quadros da realidade brasileira e paulista. Resultados: modelos diferentes de organização convivem na APS, Ações Programáticas (1970) e Saúde da Família (1996). Resultados ligeiramente mais positivos na Estratégia de Saúde da Família: indicadores de saúde, equipes, integralidade na abordagem, qualidade da atenção e acesso. As dificuldades apontadas foram: diferentes graus de implantação do programa e das equipes entre os municípios; visitas domiciliares apenas pelos agentes comunitários de saúde; rotatividade dos recursos humanos; dificuldades de encaminhamento para outros níveis de atenção; desigualdades entre os municípios; fragmentação e diferentes desenvolvimentos dos sistemas locais e regiões de saúde; subfinanciamento do sistema, especialmente no nível ambulatorial e de apoio diagnóstico. Conclusão: para o estabelecimento do SUS há importância estratégica das questões referentes aos recursos humanos, tais como: relação serviços e instituições de ensino, volume de profissionais e trabalhadores, distribuição, mercado de trabalho, formação, perfil, expectativas, resistência.
\end{abstract}

Palavras-chave: Atenção Primária à Saúde, Acesso aos Serviços de Saúde, Recursos Humanos.

\section{Universalization of the access to the Unified Health System in Brazil: challenges for the Primary Health Care}

\begin{abstract}
Introduction: Twenty-five years after the promulgation of the 1988 Federal Constitution in Brazil, which defined a "Sistema Único de Saúde" ('SUS', or "Unified Health System” - UHS) for the country, many advances have occurred, but many challenges still remain. Objective: To present the theoretical and practical bases of the “Atenção Primária à Saúde" ("APS', or "Primary Health Care" - PHC), in the Brazilian and Sao Paulo State experiences; to discuss the service "access" category, entrances to UHS and challenges for the universalization of the access based on PHC. Methodology: Results analysis of the researches that have investigated the different Brazilian and Sao Paulo State situations. Results: Different organization models coexist in PHC: the "Ações Programáticas" (or "Programmatic Actions" - 1970) and the "Saúde da Família" (or "Family Health" - 1996). Results that are slightly more positive appear in the "Family Health" strategy: health indicators, staff, completeness of approach, and quality of assistance and access. Difficulties: different degrees in the deployment of the program and staff in different municipalities; home visits only by CHA (community health agent); constant human
\end{abstract}

Autor para correspondência: Regina Maria Giffoni Marsiglia, Pontifícia Universidade Católica de São Paulo, São Paulo, SP, Brasil, e-mail: regimarsiglia@ig.com.br

Recebido em 23/7/2012; Aceito em 31/8/2012. 
resources (HR) shifts; difficulties in the follow-up to other levels of assistance; inequality among municipalities; fragmentation and different development levels of the local systems and health regions; under-financing of the system, especially at out-patient level and diagnosis support. Conclusion: Strategic importance of Human Resource matters: relation between services and education institutions, amount of professionals and workers, distribution, labor market, formation, profile, expectations and resistance.

Keywords: Primary Health Care, Health Services Accessibility, Human Resource.

\section{Introdução}

Decorridos quase 25 anos da promulgação da Constituição Federal de 1988 no Brasil, que definiu um Sistema Único de Saúde - SUS para o país, de caráter público, universal, descentralizado e hierarquizado, pautado pelos princípios da integralidade na atenção, equidade no atendimento e sob controle social da sociedade, é possível avaliar que houve avanços, mas que permanecem grandes desafios a serem enfrentados.

A implantação do SUS de 1990 a 2012, se de um lado vem demonstrando tendência à universalização, de outro aponta para a configuraçáo de um sistema dual, fragmentado, diferenciado, desintegrado, sem equidade e com dificuldades de acesso, ou acesso limitado, para segmentos importantes da população. É importante considerar que $25 \%$ da população - trabalhadores do mercado formal, público ou privado, famílias de maior poder aquisitivo e faixas mais jovens da população - recorrem ao Sistema Suplementar de Assistência, e não ao SUS, para o atendimento de parte considerável de suas necessidades de atenção. Porcentagem esta que tende a aumentar em regiōes e municípios de maior desenvolvimento industrial e econômico.

A proposta de ampliação da Atenção Primária à Saúde - APS, sob diferentes denominaçóes e abordagens, vem se desenvolvendo no país há várias décadas, e, a partir da implantação do SUS, tem sido apontada como porta de entrada e acesso da população que se utiliza do SUS aos serviços de saúde nos vários níveis de complexidade tecnológica.

Os objetivos dessa reflexão são: primeiramente, apresentar as bases teóricas e práticas internacionais da Atenção Primária à Saúde; a experiência particular do Brasil e do estado de São Paulo. Em seguida, discutir as concepçóes existentes sobre a categoria acesso na literatura e seus determinantes, bem como os desafios postos para a universalização do acesso no SUS, partindo-se das Unidades Básicas de Saúde - UBS, enquanto porta de entrada ao sistema de saúde, que deve ser integrado e integral, hierarquizado e descentralizado. E, finalizando, abordar os desafios para a Atenção Primária à Saúde, com particular atenção para as questôes decorrentes dos recursos humanos inseridos nos serviços de saúde.

\section{Atenção Primária à Saúde no âmbito internacional}

O primeiro documento que anunciou alguns dos princípios e características do que hoje denominamos de Atenção Primária à Saúde foi apresentado em 1920, por Lord Dawson of Pen, no Reino Unido, com a finalidade de organizar os serviços de saúde e torná-los disponíveis para a população que residia em um determinado território, tendo ficado conhecido com Relatório Dawson. O autor propôs que centros de saúde organizassem a assistência à saúde, que deveria ser regionalizada e hierarquizada, definindo-os como:

[...] uma instituição equipada para serviços médicos preventivos e curativos a serem prestados sob condução de médicos generalistas (general practitioners - GPs) do distrito, em cooperação com serviços de enfermagem eficientes e apoio de especialistas [...] (LUPPI et al., 2011, p. 335).

Essa concepção influenciou a criação do Sistema Nacional de Saúde inglês, em 1948, quando do início do desenvolvimento das experiências das sociais democracias, através dos Estados de Bem-Estar Social, em vários países do mundo.

Trinta anos depois, a Conferência Internacional sobre Cuidados Primários de Saúde, realizada em 1978 na cidade de Alma-Ata na União das Repúblicas Socialistas Soviéticas - URSS, firmou a noção de Atenção Primária à Saúde - APS. E a Organização Mundial da Saúde - OMS incentivou os países a desenvolverem seus sistemas de saúde, na perspectiva de prestar atenção ao conjunto da população, a partir da APS, visando cumprir a meta de Saúde para Todos no ano 2000.

Ao final dos anos 2000, a OMS reafirmou a necessidade de se instituírem sistemas de atenção à saúde, com base em uma APS bem desenvolvida e resolutiva, utilizando a concepção de cuidados 
primários à saúde, atuando na interface entre a população e o sistema de saúde, tendo em vista a promoção da saúde, prevenção da doença, assistência e cuidados em geral (ORGANIZAÇÃO..., 2008).

A Atenção Primária à Saúde remete-nos à ideia de uma atenção não especializada, de baixa densidade tecnológica, em unidades de saúde descentralizadas, enquanto porta de entrada dos usuários a um sistema hierarquizado, segundo diferentes níveis de complexidade tecnológica.

Para muitos países, a Atenção Primária à Saúde tem o caráter de um programa focalizado, seletivo e de oferta limitada, destinado a grupos populacionais de baixa renda, sem garantia de acesso aos outros níveis de atenção, que incorporam tecnologia de média e alta complexidade. Já em vários países europeus e no Canadá, a APS é considerada como o primeiro nível de atenção de um sistema de saúde complexo, e responsável pela coordenação do cuidado e organização do sistema (LAVRAS, 2011; CONILL, 2008).

O Observatório Europeu de Sistemas e Políticas de Saúde, analisando as reformas realizadas por países pertencentes à Uniăo Europeia, em seus sistemas de saúde, destacou a importância da Atenção Primária à Saúde nesses processos. Na mesma perspectiva, Starfield (2002) apontou os atributos necessários para as práticas de APS: primeiro contato do usuário, longitudinalidade, integralidade e coordenação. E ao analisar vários sistemas de saúde europeus e escandinavos concluiu que aqueles que se apoiam em uma base sólida e extensiva de APS para sua população obtêm melhores resultados do que aqueles que investem grande quantidade de recursos financeiros, físicos e humanos, mas não dispóem dessa sólida base de APS.

\section{Brasil}

Para Mota e Schraiber (2011, p. 838), a emergência do conceito de APS fez com que se substituísse a noção de "cuidados primários", relativa ao conteúdo de açôes técnicas, pela de "atenção primária", como um nível de atenção ou porta de entrada para um sistema hierarquizado e regionalizado de saúde, que já vinha sendo processado no Brasil, desde meados da década de 1970, pelo movimento da Reforma Sanitária.

O Programa de Interiorização das Açóes de Saúde e Saneamento - PIASS, proposto em 1976 pelo Ministério da Saúde, para municípios com até 20 mil habitantes, desenvolveu-se inicialmente em cidades do Nordeste, e depois expandiu-se para outras regióes do país. O PIASS já incorporava agentes de saúde, a participação comunitária, baseava-se em experiências de medicina simplificada e medicina comunitária.

A Sétima Conferência Nacional de Saúde, em 1980, constituiu-se em um fórum de debates para o aprofundamento da proposta de "cuidados primários de saúde", a partir do subtema "[...] serviços básicos de saúde e sua articulação com os demais níveis de atendimento [...]" e "recursos humanos" (MOTA; SCHRAIBER, 2011, p. 840), configurando-se a APS no país, com possibilidade de extensão da cobertura da assistência e, ao mesmo tempo, como porta de entrada que ordenasse o sistema de saúde, pressupostos que, posteriormente, passaram a ser contemplados no SUS, promulgado na Constituição Federal de 1988.

Em 1993, a Portaria MS no 692 propôs a implementação do Programa Saúde da Família (PSF), constituído por uma equipe mínima, composta por um médico generalista, um enfermeiro, dois auxiliares de enfermagem e cinco ou seis agentes comunitários de saúde (ACS), e também o Programa de Agentes Comunitários de Saúde (PACS).

A expansão dessas propostas ocorreu no país a partir da definiçáo da NOB SUS 01/1996, quando o Ministério da Saúde deliberou sobre a forma de financiamento da Atenção Básica à Saúde - PAB, com um componente fixo e outras variáveis, através de repasses de recursos financeiros do Fundo Nacional de Saúde para os fundos municipais de saúde, estipulando um valor fixo per capita, para ser alocado pelos municípios na Atenção Básica.

Em 1997, o PSF foi definido como Estratégia para a Atenção Básica pelo Ministério da Saúde, e, em 1998, a Portaria n 3.925 apresentou o que se entende por Atenção Básica:

[...] conjunto de açóes, de caráter individual ou coletivo, situadas no primeiro nível de atenção dos sistemas de saúde, voltadas para a promoçáo de saúde, prevençáo de agravos, tratamento e reabilitação [...] (BRASIL, 1998).

Em 2006, a portaria 648/GM apresentou uma concepção mais abrangente da Atenção Básica, aproximando-a da definição da APS, orientando que ela deveria ser desenvolvida por:

[...] meio do exercício de práticas gerenciais e sanitárias democráticas e participativas, sob a forma de trabalho em equipe, dirigidas a populaçóes de territórios bem delimitados, pelos quais assume a responsabilidade sanitária... utiliza tecnologias de elevada 
complexidade e baixa densidade, que devem resolver os problemas de saúde de maior frequência e relevância em seu território. É o contato preferencial dos usuários com os sistemas de saúde. Orienta-se pelos princípios da universalidade, da acessibilidade e da coordenação do cuidado, do vínculo e continuidade, da integralidade, da responsabilização, da humanização, da equidade e da participação social (BRASIL, 2006).

Dados apontam que, em 2007, 90\% dos municípios contavam com Equipes de Saúde da Família, com cobertura populacional de 46\%; em 2010 existiam 31 mil equipes, e cobertura populacional de 52\% (LUPPI et al., 2011, p. 341).

\section{São Paulo}

Em São Paulo, ao final dos anos 60, quando o Dr. Walter S. P. Leser assumiu a direção da Secretaria de Estado da Saúde (SES-SP), deu início a uma importante reforma administrativa, que unificou vários programas verticais de atenção à criança, mulher, saúde mental, hanseníase e tuberculose, e os inseriu em um Centro de Saúde, sob a direção única de novos médicos sanitaristas, com cursos de especialização na Faculdade de Saúde Pública da USP e em outras instituiçôes de ensino da área da saúde. Terminados os cursos de especialização, esses profissionais prestavam concurso público para a carreira de médico sanitarista da SES-SP.

Ao mesmo tempo, a reforma administrativa dividiu a Secretaria de Saúde em quatro grandes coordenadorias: de Saúde da Comunidade, Saúde Mental, Administração Hospitalar e Serviços Técnicos Especializados. Os centros de saúde passaram a integrar a Coordenadoria de Saúde da Comunidade e desenvolver atividades de programas que nas décadas passadas eram verticalizados; os centros de saúde passaram a ser organizados sob o modelo da Programação em Saúde.

A prática nos centros de saúde era dividida em: atividades de rotina e eventuais, e a assistência medico-sanitária avançou, superando-se

[...] no terreno das práticas a defesa majoritária dos centros de saúde como exclusivamente para açóes preventivas, dualismo já superado no discurso acadêmico desde os anos 1950 [...] (MELLO; IBAÑEZ, VIANA, 2011, p. 859).

Schraiber, Nemes e Mendes-Gonçalves (2000, p. 48-65) e Nemes (1990, p. 65-116) apontam diferenças entre a proposta da Programaçáo em Saúde implantada em Sáo Paulo nos centros de saúde na década de 1970, que ampliou e diversificou a assistência médica individual, junto com a extensão de cobertura. E a proposta denominada de Ação Programática em Saúde, lançada dez anos depois, que buscou aprimorar o modelo da programação: na perspectiva de considerar as determinaçóes sociais do processo saúde-doença, as construçóes interdisciplinares na abordagem do indivíduo, da família e da comunidade, no diagnóstico de saúde, na demanda pelos serviços, na melhoria da comunicação serviço-população e participação dos usuários.

Já a implantação do PSF em Sáo Paulo começou em 1996, tendo a SES-SP decidido por uma política estadual baseada em três pontos, segundo Guedes, Santos e Di Lorenzo (2011, p. 877-879):

- A SES-SP prestaria apoio técnico aos municípios para esse processo. Em 2002 havia 1.638 equipes distribuídas entre 402 municípios, com cobertura de 5,6 milhóes de pessoas, isto é, $20 \%$ da população;

- A SES-SP passou a financiar, a partir de 1999, 2/3 do custeio das equipes de PSF em municípios considerados prioritários, dadas as péssimas condiçôes de saúde e as dificuldades de acesso da população desses municípios ao sistema de saúde, o que incluía municípios com baixo Índice de Desenvolvimento Humano (IDH), as áreas de assentamento e as comunidades remanescentes de quilombolas nas regiôes do Vale do Paraíba e litoral Norte, Vale do Ribeira e região de Itapeva - Itararé, regiáo Oeste, Unipontal; e

- A SES-SP passou a desenvolver um projeto no município da capital, com apoio do Ministério da Saúde e algumas entidades de caráter filantrópico, como a Casa de Saúde Santa Marcelina (e, posteriormente, a Fundação Zerbini, a Universidade de Santo Amaro e a Associação Congregação de Santa Catarina), denominada de Projeto Qualidade Integral em Saúde (Qualis), porque a secretaria municipal de São Paulo estava implantando um sistema de forte caráter privado, o Plano de Assistência à Saúde (PAS), baseado em cooperativas de serviços. A experiência chegou a contar com 218 equipes e permaneceu até 2002 , quando na mudança política da administração municipal a SMS-SP aderiu ao SUS e a Estratégia do PSF recebeu essas equipes Qualis e expandiu sua implantação através de convênios com 12 instituiçôes parceiras. 


\section{Categoria acesso}

A palavra acesso vem sendo bastante utilizada nas políticas públicas, especialmente na área de saúde. No entanto, como é um conceito complexo e multideterminado, tem levado a imprecisóes e polissemias, o que torna necessário discuti-lo (CARNEIRO JUNIOR; MARSIGLIA, 2009).

Para haver acesso, a primeira condição é a existência de serviços em quantidade e qualidade, isto é, depende da disponibilidade de serviços: localização, distribuiçáo geográfica e no território, de acordo com as necessidades da população local.

Outra dimensão da categoria acesso é a acessibilidade: organização, planta física, recursos humanos em quantidade e qualidade adequadas, horário de funcionamento, acolhimento, capacidade de adaptaçâo às necessidades diferenciadas dos usuários, preocupação com o estabelecimento de equidade, rotinas e protocolos preocupados com a inclusão, valorização das tecnologias leves de trabalho (escuta, vínculo etc.).

E também a aceitabilidade que apresenta: grau de satisfação do usuário, ser legitimado e aceito pela populaçáo, de acordo com suas experiências culturais anteriores, e o estabelecimento de novos consensos através da participação social (ADAY; ANDERSON, 1974; PENCHANSKY; THOMAS, 1981; FRENK, 1992).

Cohn et al. (2002) resumiram essas dimensões da categoria acesso com a seguinte definição:

[...] a concepção de acesso oculta a essência de uma realidade que, em si mesma, é uma totalidade complexa e indivisível. Há, embutidas no conceito de acesso, noções de presença física de recursos, eficácia ou resistência na capacidade de produzir serviços úteis do lado de quem planeja e organiza; e há, do lado daqueles para quem são idealizados e operacionalizados, o reconhecimento de obstáculos, mas também capacidade de avaliação [...] (p. 81).

Penchansky e Thomas (1981) chamam atenção ainda para duas outras dimensóes: acomodação, enquanto satisfaçáo do usuário com a organização e atendimento que recebe, e capacidade econômica, especialmente quando há pagamento pelos serviços prestados, as características dos planos de pagamento, a possibilidade dos usuários arcarem com essas despesas. No caso do Brasil, podemos exemplificar com o acesso aos serviços da Saúde Suplementar, já que parte dos usuários pessoalmente, ou alguém de sua família, usa os dois sistemas de saúde existentes no país, o público e o privado.

\section{Atenção Primária à Saúde: "porta de entrada" ao sistema de saúde no país}

A questão da "porta de entrada" ao sistema de saúde também é objeto de discussão: algumas concepçôes defendem a entrada dos usuários apenas através dos serviços de APS, apoiando-se nas proposiçóes da Conferência de Alma - Ata (1978). Apontam a APS como potencialmente viabilizadora de cobertura, racionalização e organização do sistema e da circulação dos usuários no interior do sistema.

Outros autores (GIOVANELLA; FLEURY, 1996; CECÍLIO, 1997; ALMEIDA; GIOVANELLA; MATTOS, 2002; TRAVASSOS et al. 2000; BOUSQUAT, 2001) criticam a concepção de sistema de saúde que eles denominam de "sistema piramidal", que apresenta a APS como a única "porta de entrada". Propóem que se observe o movimento e a demanda espontânea da população, que muitas vezes procura os serviços de pronto atendimento ou de emergência para adentrar no sistema.

Do nosso ponto de vista, é menos importante definir por onde, ou por que "porta" a população deve entrar no sistema: o mais importante é que ele esteja organizado, articulado em seus vários níveis de complexidade, e que uma vez tendo adentrado no sistema de saúde, o usuário possa circular e transitar pelos serviços das redes loco- regionais, de um nível para outro, enquanto demanda orientada tecnicamente, de acordo com a necessidade de seu caso.

\section{Desafios}

Como bem observa Lavras (2011):

[...] mesmo reconhecendo os avanços na organização da APS no Brasil, desde o início do processo de desenvolvimento do SUS, há que se reconhecer que grandes entraves devem ser superados para que os propósitos explícitos na política nacional possam ser cumpridos e para que possa desempenhar seu papel de organizadora do sistema e coordenadora do cuidado em saúde. (p. 871).

O maior entrave reside no financiamento insuficiente do sistema de saúde, que deve ser assumido pelas administraçóes públicas dos três níveis: municipal, estadual e federal. A discussão dessa questão vem se arrastando há mais de uma década com uma proporção desigual de investimento entre as esferas do governo. Enquanto os municípios, 
no geral, ampliaram a participação da saúde no seu orçamento, destinando ao setor, pelo menos, $15 \%$ de suas receitas, os governos estaduais repassando $12 \%$ para a saúde, o outro lado, a União, tem destinado no máximo $4 \%$.

De acordo com a Proposta de Emenda Constitucional - PEC 29, a União deveria ir aumentando progressivamente a destinação de recursos para o SUS, até chegar a $10 \%$ de sua receita. A proposta não foi aprovada no nível federal em 2012, sendo que a União defendia a necessidade de aprovar, novamente, a Cobrança Provisória por Movimentação Financeira (CPMF) para financiamento do sistema. Portanto, este ainda permanece com um grande desafio. Vale lembrar que o nível secundário tem apresentado a pior situação de subfinanciamento, o que leva ao estrangulamento do sistema, sem dúvida.

Por outro lado, várias pesquisas de avaliação sobre a APS no Brasil, realizadas desde 2008, em várias regiōes, dentre as quais destacamos as contribuiçóes de Felisberto et al. (2002), Conill (2002), Goulart (2004), Mascarenhas (2003), Cavalcante et al. (2006), Castro et al. (2007) e Canesqui e Spinelli (2008) apontaram resultados positivos da implantação do PSF no comportamento dos indicadores de saúde, relacionamento das equipes, avanço na integralidade, custos menores da atençáo, melhor oferta de serviços, qualidade e acesso da população, quando comparado com as experiências e modelos anteriores da APS.

No entanto, várias dificuldades também foram identificadas, dentre elas destacam-se: diferentes graus de implantação do programa e das equipes; realização das visitas domiciliares em geral, apenas pelos ACS, sem envolvimento dos outros membros das equipes; dificuldades de encaminhamento dos usuários para outros níveis de atenção, comprometendo a integralidade da proposta; falta de capacitação dos recursos humanos envolvidos; dificuldade de composição das equipes ou alta rotatividade, especialmente de médicos (MARSIGLIA, 2012).

Questiona-se também sobre qual será o futuro da APS e do sistema de saúde como um todo: se ele manterá a tendência de organização piramidal, com pelo menos três níveis de atençâo, ou, se no futuro, só teremos dois níveis de atenção: o hospitalar para os casos mais graves e que necessitam de cuidados constantes, e o não hospitalar, incluindo o que hoje denominamos de APS, o pronto atendimento, os ambulatórios de especialidade e a assistência domiciliar. O futuro dirá.

Uma experiência a destacar é a do município de Barueri, na Grande São Paulo, que não desenvolveu a Estratégia de Saúde da Família, contando com um número reduzidíssimo de equipes, mas que tem 15 UBS, dentre as quais: 11 funcionam até as $20 \mathrm{ou}$ 21 horas diariamente, há unidades que contam com especialidades médicas, cardiologia, oftalmologia, dermatologia, bem como com alguns exames complementares, que em geral não se encontram em serviços de APS: eletrocardiograma, colposcopia, prótese dentária, e outros, de certa forma é como se fundisse a APS com o ambulatório de especialidades e o pronto atendimento no mesmo espaço físico (SILVA, 2012). Seria esta uma forma "deturpada" de APS ou o seu futuro?

Algumas vantagens a favor da Estratégia de Saúde da Família apareceram na comparação entre as UBS organizadas sob o modelo da Ação Programática e as do modelo do PSF: acesso mais fácil, menor tempo para marcação de consultas, prontuários organizados por família, orientação comunitária. Já os vínculos entre usuários, famílias e profissionais apresentam-se mais acentuados em ambos os modelos, com ligeiro destaque para os de Saúde da Família (SALA et al., 2011).

Dentre os desafios, constituem importantes obstáculos para a universalização do sistema o tamanho, a diversidade e a desigualdades entre os municípios; a fragmentação, diferentes desenvolvimentos dos sistemas locais e regiōes de saúde com diversos níveis de incorporação tecnológica; as condições de vida e trabalho, o perfil epidemiológico, demográfico e necessidades de saúde dos diferentes segmentos que compóem a população.

Quanto à realidade do acesso, através da APS, apesar da expansão, encontram-se ainda falhas na disponibilidade dos serviços, tanto do ponto de vista de quantidade quanto de localização, nem sempre adequada, e dificuldades com o sistema de transporte.

$\mathrm{Na}$ acessibilidade: existem formas inadequadas de funcionamento, fluxos interrompidos, rotinas e estratégias para conter ou contornar a demanda, registros inadequados ou incompletos de informaçôes sobre o usuário e sua família; falta ou inadequação de recursos físicos, financeiros e humanos; falta de planejamento e gerência nos serviços: espera excessiva para marcaçáo de consultas e procedimentos, resultados de exames, definição de terapêutica a ser seguida, poucas atividades educativas, com e na comunidade.

$\mathrm{Na}$ aceitabilidade: recursos humanos despreparados, resistentes à APS, relações de trabalho precárias, rotatividade de pessoal, individualismo, despreparo para o trabalho em equipe, não disponibilidade para desenvolver vínculo com os 
usuários, abordagem fragmentada, náo integral do usuário e suas famílias, despreparo para a abordagem de famílias, dificuldades para encaminhamento dos usuários para outros níveis.

E por parte dos usuários, a não aceitação daquele nível de atenção, valorização da incorporação de tecnologia e especialização, não aceitação do atendimento realizado por outros profissionais, que não médicos. Não aceitação das visitas domiciliares, sensação de invasão de sua privacidade, especialmente de famílias pertencentes às camadas médias, que possuem convênios e têm acesso ao Sistema Suplementar de Atenção; não planejamento democrático, cumprimento de programas verticais, não adequação das propostas às particularidades do território e da população que ali vive ou trabalha.

Destacamos, ainda, em particular, as questóes referentes aos recursos humanos que se inserem no sistema de saúde: volume, distribuição, mercado de trabalho, formação, perfil, expectativas. Um dos entraves advém da valorizaçáo cultural da especialização e incorporação tecnológica, tanto pela sociedade, instituiçóes de ensino e profissionais de saúde, isto é, das "tecnologias duras" de trabalho, em detrimento das "tecnologias duras-leves" ou "tecnologias leves" (MERHY, 2002) cujos conhecimentos, habilidades de utilização são mais requeridas na APS, mas que no imaginário dominante são mais simples e pouco importantes.

O estabelecimento de consensos a respeito do trabalho em equipe, especialmente na APS, retira do médico a exclusividades ou mesmo a centralidade das decisóes, ao mesmo tempo que pode refletir positivamente na demanda da população, que tradicional e historicamente no país tende a buscar fundamentalmente a consulta médica, relegando as atividades desenvolvidas pelos outros profissionais a segundo plano e dificultando a efetivação da integralidade da atenção.

$\mathrm{Na}$ composição das equipes multiprofissionais na APS, ainda predominam médicos, enfermeiros e cirurgiôes-dentistas. Pesquisa realizada em 87 UBS da regiáo norte e da área central da cidade de São Paulo, em 2007, apontou as seguintes porcentagens na amostra analisada: $17,4 \%$ eram médicos, outros $17,4 \%$ enfermeiros, em seguida estavam os cirurgiôes-dentistas. Outras profissóes foram bem menos frequentes: assistentes sociais, psicólogos, nutricionistas; e, menos comuns ainda, não existindo na maioria das UBS farmacêuticos, fonoaudiólogos, fisioterapeutas e terapeutas ocupacionais. Essa situação se revela especialmente preocupante, face ao perfil epidemiológico e à complexidade cada vez maior das situaçôes que vêm sendo atendidas na APS. Do ponto de vista das ocupaçóes de nível técnico, essa pesquisa revelou ainda, que: $17,6 \%$ eram auxiliares de enfermagem, $14 \%$ pertenciam à área administrativa e $7,1 \%$, eram agentes comunitários de saúde (MARSIGLIA, 2011).

Por outro lado, experiências e práticas de relaçóes mais horizontalizadas no cotidiano do trabalho entre os profissionais, entre os trabalhadores entre si; os vínculos estabelecidos com os usuários; o conhecimento profundo e compartilhado sobre a realidade local e prioridades a serem atendidas são essenciais para a efetivaçáo da Atenção à Saúde no nível primário da atenção.

Já o estabelecimento de protocolos de atendimento, acordados entre a APS e os outros níveis de atenção, nos sistemas locais e regionalizados, possibilita a implantação de uma atenção compartilhada, com reconhecimento da importância e contribuição específica de cada nível de atenção, considerando-se a linha de cuidado estabelecida para cada usuário do sistema; e assegura o acesso universal dos diferentes segmentos da população aos vários níveis de complexidade e resolutividade possível no sistema de saúde.

Outra questão relevante a respeito dos recursos humanos para a APS é que tanto os profissionais quanto os trabalhadores, em geral, necessitam de uma formação adequada para desenvolver suas atividades nesse nível da atenção, desde sua formação básica de nível superior ou técnica ocupacional, bem como participar de processos contínuos de educação permanente. Esse aspecto tem exigido uma aproximação constante das administrações públicas de saúde de todos os níveis, com as universidades e instituições de ensino da área de saúde, a criação de Centros de Formação de RH (Cefor), de Escolas Técnicas do SUS (ETSUS) e a implantação de espaços de articulação permanente entre instituições de ensino e serviços de saúde, como os Polos de Capacitação (1998-2002), Polos de Educação Permanente (PEP) (2002-2008), e mais recentemente as Comissóes de Integração Ensino-Serviços (CIES) (2009-2012) (BATISTA; GONÇALVES, 2011).

Dentre as experiências de apoio aos municípios, é fundamental que se estabeleça o papel das secretarias estaduais, além do Ministério da Saúde. A experiência da SES-SP vem mostrando a importância de se auxiliar os gestores municipais na qualificação dos serviços de APS, através do Programa de Articuladores de Atenção Básica, com prioridade para municípios de menos de 100 mil habitantes, que no estado de São Paulo somam 573 dentre os 645 existentes. Cada articulador tem como responsabilidade apoiar 10 municípios desse porte, localizados na mesma 
região de saúde: "A atuação dos articuladores nos municípios deve ser orientada para o apoio ao desenvolvimento da integração entre a gestão do sistema loco-regional e o gerenciamento das ações de assistência nas unidades de atenção primária" (ANDRADE; CASTANHEIRA, 2011, p. 985).

\section{Referências}

ADAY, L. A.; ANDERSON, R. A framework for the study of access to medical care. Health Services Research, Ann Arbor, v. 9, n. 3, p. 208-220, 1974.

ALMEIDA, P. F.; GIOVANELLA, L.; MATTOS, M. Sistema de saúde brasileiro: dilemas da universalização. Saúde em Debate, Rio de Janeiro, v. 26, n. 61, p. 137-154, maio/ago. 2002.

ANDRADE, M. C.; CASTANHEIRA, E. R. L. Cooperação e Apoio Técnico entre Estado e Municípios: a experiência do Programa Articuladores da Atenção básica em São Paulo). Saúde e Sociedade, São Paulo, v. 20, n. 4, p. 980- 990, out./dez. 2011. http://dx.doi.org/10.1590/ S0104-12902011000400015

BATISTA, K. B. C.; GONÇALVES, O. S. J. Formação dos Profissionais de Saúde para o SUS: significado e cuidado. Saúde e Sociedade, São Paulo, v. 20, n. 4, p. 884-8 99, out./dez. 2011.

BOUSQUAT, A. Conceitos de espaço na análise de políticas de saúde. Lua Nova, São Paulo, n. 52, p. 71-92, 2001. http://dx.doi.org/10.1590/S0102-64452001000100004

BRASIL. Ministério da Saúde. Saúde da Família: uma estratégia para reorganização do modelo assistencial. Brasília: Ministério da Saúde, 1998.

BRASIL. Ministério da Saúde. Portaria GM no 648, de 28 de março de 2006. Política Nacional de Atenção Básica. Revisão das diretrizes e normas para organização da Atenção Básica para o PSF e o PACS. Diário Oficial da República Federativa do Brasil, Poder Legislativo, Brasília, DF, 04 abr. 2006. Seção 1.

CANESQUI, A. M.; SPINELLI, M. A. S. A implementação do Programa de Saúde em municípios do estado de Mato Grosso, Brasil. Cadernos de Saúde Pública, Rio de Janeiro, v. 24 no. 4, p. 862-870, abr. 2008.

CARNEIRO JUNIOR, N.; MARSIGLIA, R. M. G. O PSF e os Princípios do SUS: disponibilidade, acessibilidade e aceitabilidade do PSF em áreas metropolitanas. In COHN, A. (Org.). Saúde da Família e SUS: Convergências e dissonâncias. Rio de Janeiro: Beco do Azougue; São Paulo: CEDEC, 2009. p. 93-112

CASTRO, J. D. et al. O Custo- Efetividade: uma comparaçáo entre o modelo "tradicional" e o modelo do Programa de Saúde da Família. Revista Brasileira de Medicina de Família e de Comunidade, Rio de janeiro, v. 3, n. 10, p. 73-144, jul./set. 2007.

CAVALCANTE, M. G. S. et al. Análise de Implementação das Estratégias de Atenção Básica nas Equipes de Saúde da Família em Município de uma região metropolitana do nordeste brasileiro. Revista Brasileira de Saúde Materno
Infantil, Recife, v. 6, n. 4, p. 437-445, out./dez. 2006. http://dx.doi.org/10.1590/S1519-38292006000400011

COHN, A. et al. A Saúde como Direito e como Serviço. 5. ed. Sáo Paulo: Cortez, 2002.

CECÍLIO, L. C. O. Modelos assistenciais em saúde: da pirâmide ao círculo, uma possibilidade a ser explorada. Cadernos de Saúde Pública, Rio de Janeiro, v. 3, n. 13, p. 469-478, jul./set.1997. http://dx.doi.org/10.1590/ S0102-311X1997000300022

CONILL, E. M. Ensaio histórico-conceitual sobre a Atenção Primária à Saúde: desafios para a organização de serviços básicos e da Estratégia Saúde da Família em centros urbanos no Brasil. Cadernos de Saúde Pública, Rio de Janeiro, v. 24, p. S7-S27, 2008. Suplemento 1.

FELisberto, E. et al. Avaliação do Processo de Implantação da Estratégia de Ação Integrada às Doenças Prevalentes na Infância no Programa de Saúde da Família no Estado de Pernambuco - Brasil. Cadernos de Saúde Pública, Rio de janeiro, v. 18, n. 6, p. 1737-1745, nov./dez. 2002.

FRENK, J. El concepto y la medicino de accesibilidad. In: WHITE, K. L. (Ed.). Investigaciones sobre servicios de salud: una antologia. Washington: OPS, 1992. p. 929-943.

GIOVANELLA, L.; FLEURY, S. Universalidade da atenção à saúde: acesso como categoria de análise. In: EIBENSCHUTZ, C. (Ed.). Politica de saúde: o público e o privado. Rio de Janeiro: Fiocruz, 1996. p. 177-198.

GOULART, F. A. A. Círculos Virtuosos e Boas Práticas em Saúde da Família. In: FERNANDES, A. S.; SECLENPALCIN, J. A. (Orgs.). Experiências e Desafios da Atenção Básica e Saúde da Família: o caso do Brasil. Brasília: OPAS, 2004.

GUEDES, J. S.; SANTOS, R. M. B.; DI LORENZO, R. A. V. A Implantação do programa de Saúde da Família (PSF) no Estado de São Paulo (1995-2002). Saúde e Sociedade, São Paulo, v. 20, n. 4, p. 875-883, out./dez. 2011. http://dx.doi.org/10.1590/S0104-12902011000400006

LAVRAS, C. Atenção primária à Saúde e a organização de Reds regionais de Atenção à Saúde no Brasil. Saúde e Sociedade, São Paulo, v. 20, n. 4, p. 867-874, out./dez. 2011. http://dx.doi.org/10.1590/S0104-12902011000400005 LUPPI, C. G. et al. Atenção Primária à Saúde/Atenção Básica. In: IBAÑEZ, N.; ELIAS, P. E. M.; SEIXAS, P. H. (Org.). Politica e Gestão Pública em Saúde. São Paulo: Hucitec Editora, 2011. p. 332-353.

MARSIGLIA, R. M. G. Perfil dos Trabalhadores da Atenção Básica em Saúde no Município de São Paulo: região norte e central da cidade. Saúde e Sociedade, São Paulo, v. 20, n. 4, p. 900-911, out./dez. 2011. http:// dx.doi.org/10.1590/S0104-12902011000400008

MARSIGLIA, R. M. G. Atenção Básica à Saúde em São Paulo: profissionais, instituiçôes parceiras, usuários. In: SILVA, J. A.; SEIXAS, P. H.; MARSIGLIA, R. M. G. Coletânea Observa RHSP: Estudos e Projetos. Sáo Paulo: CEALAG, 2012. Eixo Recursos Humanos na Atenção Básica à Saúde - v. 2. p. 23-89. No prelo.

MASCARENHAS, M. T. M. Avaliando a Implantação da Atenção Básica em Saúde no Município de Niterói/RJ: 
Estudos de Caso em UBS e Módulo do programa Médico de Família. 2003. 181 f. Tese (Doutorado em Saúde Pública)-Escola Nacional de Saúde Pública, Fundação Oswaldo Cruz, Rio de Janeiro, 2003.

MELLO, G. A.; IBAÑEZ, N.; VIANA, A. L. Um Olhar Histórico sobre a Questão Regional e os Serviços Básicos de Saúde no Estado de São Paulo. Saúde e Sociedade, São Paulo, v. 20, n. 4, p. 853-866, out./dez. 2011. http:// dx.doi.org/10.1590/S0104-12902011000400004

MERHY, E. E. Saúde: a cartografia do trabalho vivo. 2. ed. São Paulo: HUCITEC, 2002. (Saúde em Debate, n. 145). MOTA, A.; SCHRAIBER, L. B. Atenção primária no Sistema de Saúde: debates paulistas numa perspectiva histórica. Saúde e Sociedade, São Paulo, v. 20, no. 4, p. 837-852, out./dez. 2011. http://dx.doi.org/10.1590/ S0104-12902011000400003

NEMES, M. I. B. Ação Programática em Saúde: recuperação histórica de uma política de programação. In: SCHRAIBER, L. B. (org. ). Programação em saúde hoje. São Paulo: Hucitec, 1990. p. 65-116.

ORGANIZAÇÃO MUNDIAL DE SAÚDE - OMS. Relatório Mundial da saúde 2008: Cuidados Primários de Saúde - Agora mais que nunca. Genebra: OMS, 2008.
PENCHANSKY, R.; THOMAS, J. W. The concept of access: definition and relationship to consumer satisfaction. Medical Care, Philadelphia, v. 12, n. 2, p. 127-140, 1981.

SALA, A. et al. Integralidade e Atenção Primária à Saúde: avaliação na perspectiva dos usuários de unidades de saúde do município de São Paulo. Saúde e Sociedade, São Paulo, v. 20, n. 4, p. 948-960, out./dez. 2011. http://dx.doi. org/10.1590/S0104-12902011000400012

SCHRAIBER, L. B.; NEMES, M. I. B.; MENDESGONÇALVES, R. B. (Orgs.). Saúde do Adulto: programas e açóes da unidade básica. 2. ed. São Paulo: Hucitec, 2000.

SILVA, E. C. Universalidade da Atenção à Saúde no SUS: o caso de Barueri - São Paulo. 2012. 247 f. Dissertação (Mestrado em Serviço Social)-Pontifícia Universidade Católica de São Paulo, São Paulo, 2012.

STARfield, B. Atenção Primária: equilíbrio entre necessidades de saúde, serviços e tecnologia. Brasília: UNESCO; Ministério da Saúde, 2002.

TRAVASSOS, C. et al. Desigualdades geográficas e sociais na utilização de serviços de saúde no Brasil. Ciência \& Saúde Coletiva, Rio de Janeiro, v. 5, n. 1, p. 133-149, 2000. http://dx.doi.org/10.1590/ S1413-81232000000100012 